\title{
Topical delivery of ebselen encapsulated in biopolymeric nanocapsules: drug repurposing enhanced antifungal activity
}

\author{
Anna Jaromin*,1, Robert Zarnowski², Magdalena Piętka-Ottlik ${ }^{3}$, David R Andes² \& Jerzy \\ Gubernator $^{1}$ \\ ${ }^{1}$ Department of Lipids \& Liposomes, Faculty of Biotechnology, University of Wroclaw, Joliot-Curie 14a, 50-383 Wroclaw, Poland \\ ${ }^{2}$ Department of Medicine, Section of Infectious Diseases, 5225 Microbial Sciences Building, 1550 Linden Dr, University of \\ Wisconsin-Madison, Madison, WI 53706, USA \\ ${ }^{3}$ Department of Organic \& Pharmaceutical Technology, Faculty of Chemistry, Wroclaw University of Science \& Technology, \\ Wybrzeze Wyspianskiego 27, 50-370 Wroclaw, Poland \\ *Author for correspondence: anna.jaromin@uwr.edu.pl
}

Aim: Ebselen (Eb) is an example of a repurposed drug with poor aqueous solubility which requires sophisticated delivery system such as nanoencapsulation in nanocapsules for topical application. Materials \& methods: Eb-nanocapsules were examined for morphology, activity against Candida spp., cytotoxicity and skin permeation. Results: Eb-nanocapsules were active against skin-infecting Candida tropicalis, Candida albicans and Candida parapsilosis yeasts (minimal inhibitory concentration values were about 4-, 2and 1.25-times lower vs free Eb, respectively) and able to suppress induced lipid oxidation in the oil/water emulsion. Moreover, demonstrated minimal toxicity in normal human dermal fibroblast cell line, whereas ex vivo skin permeation studies showed no transdermal passage and strong interactions with stratum corneum. Conclusion: Eb-nanocapsules represent a promising, safe and complementary alternative to the treatment of cutaneous candidiasis.

First draft submitted: 31 October 2017; Accepted for publication: 2 February 2018; Published online: 6 June 2018

Keywords: alginate $\bullet$ antifungal activity $\bullet$ Candida $\bullet$ drug repurposing $\bullet$ ebselen $\bullet$ fungal infection $\bullet$ nanocapsules - organoselenium compound

Ebselen (2-phenyl-1,2-benzisoselenazol-3(2H)-one) (Eb) is an example of multifunctional already approved repurposing drug with well-characterized toxicology and pharmacology. This organoselenium compound is known to be clinically safe and owing to pharmacologically desired properties is currently under clinical trials for the prevention and treatment of various disorders such as cardiovascular diseases, arthritis, stroke, atherosclerosis and cancer. Eb exhibits strong anti-inflammatory and antioxidant activity [1-3]. Moreover, the ability of this compound to mimic GPx activity makes it a potential drug for the treatment of diabetes related disorders associated with reduced GPx levels. It was shown that Eb effectively compensates for the lack of GPx and acts as an effective therapeutic molecule for the treatment of diabetes related atherosclerosis and nephropathy [4]. Other studies report that drug affects the phosphoinositide cycle and has CNS effects that may be relevant to the treatment of bipolar disorder [5] and can decrease impulsivity and produce a positive bias in emotional processing [6]. Recent studies also showed that Eb is an inhibitor of HIV-1 capsid C-terminal domain dimerization. Eb inhibits early viral postentry events of the HIV-1 life cycle by impairing the incoming capsid uncoating process [7]. Another interesting property is the activity against several microorganisms. It was proven that this organoselenium compound is a potent inhibitor of the Mycobacterium tuberculosis Ag85 complex [8]. Its robust antimicrobial activity against clinical multidrug-resistant Gram-positive pathogens, including Staphylococcus, Streptococcus and Enterococcus, but not against Gram-negative pathogens was identified. Additionally, the activity of Eb against Gram-positive pathogens exceeded those activities determined for vancomycin and linezolid, drugs of choice for recommended treatment of Enterococcus and Staphylococcus infections [9-12]. Thangamani et al. postulated that Eb has a great potential for topical treatment of multidrug-resistant staphylococcal infections and certain yeast infections $[11,13]$. 
The incidence of opportunistic infections has been on the rise over the last few decades. The prevalence of this type of disease has been increasing mainly due to immunosuppressive treatments following transplantation procedures or as results of serious metabolic, hematological or immunological diseases. Opportunistic superficial yeast infections are diseases caused by saprophytic fungi that usually are asymptomatic in healthy host, but they primarily develop when the immune system is weakened. Other conditions leading to opportunistic superficial mucosal infections include disadvantageous weather conditions, inappropriate clothing, poor hygiene, or obesity in general the conditions which create warm, moist and poorly ventilated areas of the skin. Infections of this tissue caused by Candida yeasts, the most common fungal pathogens in humans, can usually be treated with a handful of topical antifungals, which kill the microorganism and reduce the spread of the infection. This approach is vital for keeping Candida yeasts at bay and avoiding their further spread into life-threatening invasive systemic-type infections [14].

There is only a handful of known antifungals readily available these days. A primary reason is that most of discovered antifungals are not only active in vivo versus pathogenic fungi, but also exhibit severe toxicity to hosts of those pathogens. Therefore the need for effective antifungal agents, especially against Candida spp., that possess low toxicologic properties continues unabated [15]. While discovering and developing a brand-new antifungal drug requires enormous amounts of both time and resources, drug repurposing is therefore one of the crucial antifungal drug advancing strategies these days that dramatically decrease obliged time frame and costs, while improving success rate. In fact, Eb as part of the National Institutes of Health Clinical Collection was one of the first repositioned bioavailable drugs considered clinically safe, but without proven use [16]. This drug represents a potent lithium mimetic as a treatment for bipolar disorder [17], however it may be potentially used in treatments of Candida spp. infections [13].

Armed with all this knowledge, we decided to develop a topical delivery system for Eb with profound antifungal efficacy arrays for prospective treatment of skin infections caused by Candida yeasts. Nanosystems are already known to be successful carriers of selenium dedicated for the inhibition of bacterial or fungal growth [18-20]. We focused on nanocapsules prepared from alginate, a natural biopolymer extracted from brown seaweed with well-known properties such as biocompatibility, biodegradability and nontoxicity, which overall makes it a great encapsulation material [21]. This anionic copolymer composed of glucuronic and mannuronic acid residues has several biomedical applications, including cell and drug delivery, dental impressions, wound dressing and bone tissue engineering [22].

In this report, we described the successful production of a series of Eb-encapsulated core-shell nanocapsules (Eb-NCS) to obtain valuable insights into novel potential therapeutic applications of Eb for use as antifungal agent for the treatment of superficial Candida-related cutaneous infections.

\section{Materials \& methods \\ Materials}

Eb was synthetized by applying the method reported previously [23]. Labrafac Lipophile WL1349 and Transcutol HP were a gift from Gattefossè (Saint Priest, France). Alginic acid sodium salt from brown algae, Span 80, sorbitan sesquioleate, fetal bovine serum, bovine trypsin, calcium chloride, Nile Red and MTT (3-[4,5-dimethylthiazol2-yl]-2,5-diphenyltetrazolium bromide) were purchased from Sigma-Aldrich (Poznan, Poland). Super Refined ${ }^{\mathrm{TM}}$ Polysorbate 80 (Tween-80) was a kind gift from Croda Poland (Krakow, Poland). Candida albicans SN250, Candida tropicalis CAY2597, Candida parapsilosis CLib214 and Candida glabrata ATCC2001 strains were kindly provided by S Noble (University of California-San Francisco), R Bennett (Brown University), G Butler (University College Dublin) and B Cormack (John Hopkins University), respectively. Flaxseed oil was from Oleofarm (Wroclaw, Poland). L-glutamine, penicillin, streptomycin and amphotericin B were from Life Technologies (Warsaw, Poland). Dialysis cellulose membranes Spectra/Por ${ }^{\circledR}$ (molecular weight cut-off [MWCO] $25 \mathrm{kDa}$ ) and $0.45 \mu \mathrm{m}$ syringe filters were provided by Spectrum Laboratories Inc. (CA, USA) and Whatman (Warsaw, Poland), respectively. Normal human dermal fibroblast cell line (NHDF) and minimum essential medium eagle alpha modifications medium were purchased from Lonza (Warsaw, Poland). All chemicals and reagents not specified in the text were of analytical grade. Deionized double distilled water was used for all the experiments.

\section{Preparation of Eb-NCS}

Eb-NCS were prepared on the basis of the method proposed by Nguyen et al. [24] using ultrasound oil-in-water emulsification and gelation step with calcium ions. Typically, a water phase and an oil phase were prepared separately. A water phase was obtained by adding a sodium alginate $(0.6 \mathrm{mg} / \mathrm{ml})$ to double distilled water under magnetic 


\section{Table 1. Characterization of ebselen-unloaded and ebselen-loaded core-shell alginate nanocapsules.}

\begin{tabular}{|c|c|c|c|c|c|c|c|c|c|c|c|}
\hline \multicolumn{3}{|c|}{ Oil phase composition } & \multicolumn{4}{|c|}{ Ebselen-unloaded core-shell alginate nanocapsules } & \multicolumn{5}{|c|}{ Ebselen-loaded core-shell alginate nanocapsules } \\
\hline $\begin{array}{l}\text { Labrafac } \\
\text { lipophile } \\
\text { WL1349 (g) }\end{array}$ & Span $80(g)$ & $\begin{array}{l}\text { Transcutol } \\
\text { HP (g) }\end{array}$ & Code & $\begin{array}{l}\text { Diameter } \\
(\mathrm{nm})\end{array}$ & Pdl & $\begin{array}{l}\zeta \text { potential } \\
(\mathrm{mV})\end{array}$ & Code & $\begin{array}{l}\text { Diameter } \\
(\mathrm{nm})\end{array}$ & Pdl & $\begin{array}{l}\zeta \text { potential } \\
(\mathrm{mV})\end{array}$ & EE (\%) \\
\hline 0.417 & 0.05 & 0.083 & $\mathrm{NCS}_{1}$ & $211 \pm 2$ & $0.22 \pm 0.01$ & $-20.8 \pm 0.1$ & $\mathrm{~Eb}-\mathrm{NCS}_{1}$ & $204 \pm 1$ & $0.15 \pm 0.01$ & $-15.4 \pm 0.1$ & $45.1 \pm 2.1$ \\
\hline 0.400 & 0.05 & 0.100 & $\mathrm{NCS}_{2}$ & $198 \pm 3$ & $0.20 \pm 0.02$ & $-20.0 \pm 0.1$ & $\mathrm{~Eb}-\mathrm{NCS}_{2}$ & $194 \pm 3$ & $0.17 \pm 0.01$ & $-15.9 \pm 0.2$ & $41.3 \pm 2.7$ \\
\hline 0.375 & 0.05 & 0.125 & $\mathrm{NCS}_{3}$ & $172 \pm 2$ & $0.16 \pm 0.01$ & $-21.1 \pm 0.3$ & $\mathrm{~Eb}-\mathrm{NCS}_{3}$ & $184 \pm 1$ & $0.12 \pm 0.02$ & $-15.9 \pm 0.1$ & $38.4 \pm 0.9$ \\
\hline 0.333 & 0.05 & 0.167 & $\mathrm{NCS}_{4}$ & $166 \pm 1$ & $0.16 \pm 0.01$ & $-20.0 \pm 0.2$ & $\mathrm{~Eb}-\mathrm{NCS}_{4}$ & $179 \pm 1$ & $0.11 \pm 0.01$ & $-15.5 \pm 0.5$ & $29.3 \pm 1.5$ \\
\hline 0.250 & 0.05 & 0.250 & $\mathrm{NCS}_{5}$ & $160 \pm 1$ & $0.15 \pm 0.01$ & $-20.4 \pm 0.3$ & $\mathrm{~Eb}-\mathrm{NCS}_{5}$ & $172 \pm 1$ & $0.11 \pm 0.01$ & $-17.1 \pm 0.9$ & $26.9 \pm 1.2$ \\
\hline
\end{tabular}

stirring ( $3 \mathrm{~h}$ at room temperature). The solution was then sealed and left for $2 \mathrm{~h}$ for degassing. After filtration through a $0.45 \mu \mathrm{m}$ filter appropriate amount of Tween- 80 was added to alginate solution (Tween-80:alginate solution 1:25 w/w). The resulting mixture was submitted to homogenization in an ultrasonic water bath for $10 \mathrm{~min}$. The oil phase consisted of Labrafac Lipophile WL1349, Span 80 and Transcutol HP containing dissolved $\mathrm{Eb}(20 \mathrm{mg} / \mathrm{g})$ (composition depicted in Table 1). All components were vigorously stirred and homogenized in an ultrasonic water bath for $10 \mathrm{~min}$, yielding a clear solution. In a typical experiment, $0.55 \mathrm{~g}$ of oil phase was dispersed in $1.955 \mathrm{~g}$ of water phase, followed by the sonication with an probe-type ultrasonic cell disruptor (Misonix Inc., NY, USA) for $2 \mathrm{~min}$, giving rise a nanoemulsion. Subsequently, still under probe sonication, the entire volume $(0.5 \mathrm{ml})$ of aqueous $\mathrm{CaCl}_{2}$ solution $(0.7 \mathrm{mg} / \mathrm{ml})$ was instantaneously injected and the ultrasonication process was further continued for another $3 \mathrm{~min}$. Finally, the obtained alginate nanocapsules were purified from the nonincorporated drug and other residues by exhaustive dialysis. Dispersions were loaded into dialysis tubing (MWCO $25 \mathrm{kDa})$ and dialyzed against distilled water at room temperature. Same procedures were applied to produce control (without Eb) nanocapsules.

\section{Characterization of Eb-NCS Microscopic evaluation}

For cryo-transmission electron miscroscopy (TEM), $3 \mu \mathrm{l}$ of Eb-loaded nanocapsules (Eb-NCS 3 ) were pipetted onto a glow-discharged 200 mesh copper grid with a lacey carbon support film. Before sample application the grid was mounted on a tweezer in the Vitrobot (FEI model MarkIII, OR, USA). In an automated sequence, excess fluid was blotted off, and the grid was plunge frozen in liquid ethane. Once frozen, the grid was mounted in a precooled cryo-transfer sample holder (Gatan model 626, PA, USA), and inserted into the TEM (Hitachi Ltd, Model HT7700, Ibaraki, Japan). The samples were observed at $120 \mathrm{kV}$ acceleration voltage and the sample temperature was kept at $-170^{\circ} \mathrm{C}$.

For scanning electron microscopy (SEM), the sample of Eb-NCS 3 was allowed to adhere to a sterile coverslip placed in a 12 -well plate overnight at $4{ }^{\circ} \mathrm{C}$ prior to fixation. A total of $1 \mathrm{ml}$ of fixative $(4 \% \mathrm{v} / \mathrm{v}$ formaldehyde, $1 \%$ $\mathrm{v} / \mathrm{v}$ ) glutaraldehyde in phosphate-buffered saline [PBS]) was then added followed by incubation at $4{ }^{\circ} \mathrm{C}$ overnight. Coverslips were then washed with PBS followed by incubation for $30 \mathrm{~min}$ in $1 \% \mathrm{v} / \mathrm{v}$ osmium tetroxide at room temperature. Samples were then serially dehydrated in ethanol $(30-100 \%, v / v)$. Critical point drying was used to completely dehydrate the sample prior to palladium-gold coating. Samples were imaged on a SEM LEO 1530, with Adobe Photoshop 7.0.1 used for image compilation. For visualization of core-shell structure, $20 \mu \mathrm{l}$ of Eb-loaded nanocapsules was placed on formvar-coated TEM copper grids for $5 \mathrm{~min}$, followed by blotting on paper filter and drying on air. Specimen was observed at $20-30 \mathrm{kV}$ of acceleration voltage by means of field emission SEM (Auriga60, Carl Zeiss Microscopy GmbH, Oberkochen, Germany) using STEM detector.

\section{MALDI-ToF-MS analysis}

Samples of nanocapsules were directly deposited onto the Opti-TOF ${ }^{\mathrm{TM}} 384$ well plate (Applied Biosystems, CA, USA) and recrystalized with $0.4 \mu \mathrm{l}$ of matrix $(10 \mathrm{mg} / \mathrm{ml}$ of $\alpha$-cyano-4-hydroxycinnamic acid in acetonitrile $/ \mathrm{H}_{2} \mathrm{O} / \mathrm{TFA}$ [70/30/0.1]). Neat standard of Eb was spotted as a positive control alongside. 'On plate' water clean-up was performed to remove any salt deposition $(1 \mu \mathrm{l}$ deposited on top of crystallized sample, let sit for 
$2 \mathrm{~s}$ and removed with pipette tip). Mass spectrum was acquired on a $4800 \mathrm{MALDI}$ ToF/ToF mass spectrometer (Applied Biosystems) scanning over 100-1000 Da (focus mass $300 \mathrm{Da}$ ) or 700-4000 Da (focus mass $2093 \mathrm{Da}$ ) mass range using 1000 shots acquired from 20 randomized regions of the sample spot at 3000 intensity and $2.059 \mathrm{kV}$ detector voltage of OptiBeam ${ }^{T M}$ (Applied Biosystems) on-axis Nd:YAG laser with $200 \mathrm{~Hz}$ firing rate and 3-7 ns pulse width in positive reflectron mass mode. External calibration with peptide standards was performed to validate mass accuracy.

\section{Particle size analysis}

The sizes of the nanocapsules were measured at $25^{\circ} \mathrm{C}$ by dynamic light scattering using Zetasizer Nano-ZS (Malvern Instruments Ltd, Malvern, UK). All samples were previously diluted with distilled water in a ratio of 1:100 v/v. The polydispersity index (PdI) was obtained by the instrument's built-in software.

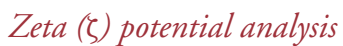

The $\zeta$ potential, reflecting the electric charge on the particle surface, was measured by the microelectrophoretic method using Zetasizer Nano-ZS. All the measurements of diluted with distilled water in a ratio of 1:100 v/v nanocapsules were performed at $25^{\circ} \mathrm{C}$.

Encapsulation efficiency

A total of $40 \mu \mathrm{l}$ of Eb-NCS was mixed with $160 \mu \mathrm{l} 0.01 \mathrm{M}$ EDTA and $1.8 \mathrm{ml}$ of methanol. Further lysis of nanocapsules and dissolution of $\mathrm{Eb}$ in methanol was achieved by stirring the samples for $60 \mathrm{~min}$. Finally, centrifugation step (13,000 rpm for $15 \mathrm{~min}$ ) was performed in order to separate the incorporated drug from the free form. The supernatant was analyzed by HPLC to determine the encapsulation efficiency (EE\%). The chromatographic system consisted of a Sunfire RP18 column $(150 \mathrm{~mm} \times 4.6 \mathrm{~mm}, 3.5 \mu \mathrm{m}$; Waters, MA, USA) and a Waters instrument (Waters 600 pump and Waters 996 UV-Vis detector). The mobile phase at a flow rate of $0.7 \mathrm{ml} / \mathrm{min}$ consisted of $1 \%$ acetic acid, methanol and propanol (25:65:10 v/v). The volume injected was $100 \mu \mathrm{l}$ and ebselen was detected at $\lambda=254$. A Millennium version 3.20 processing module was used to record and process the chromatogram. The method was linear in the range of $0.1-6 \mu \mathrm{g} / \mathrm{ml}$. The EE\% was calculated using Equation 1 .

$$
\mathrm{EE}(\%)=(\text { Actual content } / \text { Theoretical content }) \times 100
$$

(Equation 1)

\section{Stability}

The average size, PdI and $\zeta$ potential of nanocapsules were measured just after preparation and after storage at room temperature for 7,14 and 28 days.

\section{Antifungal susceptibility testing}

Nanocapsules added to Candida spp. cultures were tested in accordance with the standards in Clinical and Laboratory Standards Institute document M27-A3 [25]. The broth microdilution method employs RPMI 1640 broth medium, incubation at $35^{\circ} \mathrm{C}$ for $24 \mathrm{~h}$, and an minimal inhibitory concentration (MIC) end point criterion of complete growth inhibition (no viable growth relative to the growth of control). $\mathrm{NCS}_{3}$ were used as controls. Three indepenedent MIC determination tests involving two replicates were performed in this study.

\section{Evaluation of Eb-NCS-to-fungal cells adhesion}

A total of $1 \mu \mathrm{l}$ of $0.1 \% \mathrm{~Eb}-\mathrm{NCS}_{3}$ was added to $99 \mu \mathrm{l}$ of 24-h-old yeast cultures of either C. tropicalis or C. glabrata followed by a 15 -min-long incubation at room temperature. Yeast cultures with added nanocapsules were then collected by brief centrifugation at $3000 \mathrm{rpm}$ and washed with PBS. Pelleted cells were fixed for SEM processing as described above.

\section{Determination of inhibition of oxidation of o/w emulsion by Eb-NCS}

Emulsion was prepared according to the method described previously [26,27]. Briefly, $15 \mathrm{mg}$ of sorbitan sesquioleate was added to $50 \mathrm{mg}$ of flaxseed oil and vigorously mixed for $20 \mathrm{~min}$. Then $2 \mathrm{ml}$ of an aqueous phase, consisting of $10 \mathrm{mM}$ Tris-HCl buffer, $\mathrm{pH} 7.4$ was added. The sample was further vortexed for $15 \mathrm{~min}$ and sonicated with probetype ultrasonic cell disruptor for $15 \mathrm{~min}$. For oxidation induction, $10 \mu \mathrm{l}$ of 1:1 (v/v) buffer diluted emulsion, 
ebselen in the form of methanolic solution or encapsulated in nanocapsules $\left(\mathrm{Eb}_{-} \mathrm{NCS}_{3}\right)$ and $10 \mathrm{mM}$ Tris- $\mathrm{HCl}$ buffer, $\mathrm{pH} 7.4$ were mixed and incubated at room temperature for $10 \mathrm{~min}$. Then, $100 \mu \mathrm{l}$ of freshly prepared aqueous solution of ammonium iron (II) sulfate hexahydrate (Mohr's salt; $2 \mathrm{mM}$ ) was added (total volume of sample was $1 \mathrm{ml}$ ). All samples were incubated at room temperature for $15 \mathrm{~min}$. Flax seed oil emulsion without ebselen addition or after treatment of nanocapsules without drug $\left(\mathrm{NCS}_{3}\right)$ were included as controls. After oxidation step, samples were used for determination of thiobarbituric acid reacting substances (TBARS) [28]. A total of $2 \mathrm{ml}$ of thiobarbituric acid (TBA) reagent (15\% trichloroacetic acid, $0.25 \mathrm{~N} \mathrm{HCl}$ and $0.5 \% \mathrm{TBA}$ ) was added to each sample, mixed and heated at $100^{\circ} \mathrm{C}$ for $30 \mathrm{~min}$. After centrifugation at $13,000 \mathrm{rpm}$ for $10 \mathrm{~min}$, the absorbance of the supernatants was measured at $535 \mathrm{~nm}$. The obtained values were used for calculation of $\%$ inhibition of oxidation according to Equation 2.

Inhibition of oxidation $(\%)=[1-($ absorbance of sample $/$ absorbance of control $)] \times 100$

(Equation 2)

\section{The cytotoxic activity test}

NHDF cell culture

NHDF cell line was cultured in minimum essential medium eagle alpha modifications medium supplemented with $10 \%$ fetal bovine serum and $2 \mathrm{mM}$ glutamine, $100 \mathrm{U} / \mathrm{ml}$ penicillin, $100 \mu \mathrm{g} / \mathrm{ml}$ streptomycin, and $25 \mu \mathrm{g} / \mathrm{ml}$ amphotericin $\mathrm{B}$. The cells were cultured in a $37^{\circ} \mathrm{C}$ incubator in a humidified atmosphere containing $5 \% \mathrm{CO}_{2}$.

\section{Cell viability assay}

Cell viability was determined by the MTT method as described earlier [29]. NHDF cells were seeded in triplicate into 96-well culture plates at $10^{4}$ cells/well and incubated at $37^{\circ} \mathrm{C}$ in a humidified atmosphere and $5 \% \mathrm{CO}_{2}$ overnight. Cells, in triplicate wells, were treated with free ebselen (as methanolic solution) and ebselen-loaded alginate nanocapsules (Eb-NCS $1, \mathrm{~Eb}_{-} \mathrm{NCS}_{3}, \mathrm{~Eb}_{-} \mathrm{NCS}_{5}$ ) to obtain final concentration of $0.01,0.1$ and $1 \mu \mathrm{g} / \mathrm{ml}$, and then incubated for 24 and $48 \mathrm{~h}$. Subsequently, the medium was carefully removed from the wells, followed by addition of $50 \mu \mathrm{l}$ of dye solution $\left(0.5 \mathrm{mg} / \mathrm{ml}\right.$ of MTT salt in culture medium) and incubated at $37^{\circ} \mathrm{C}$ for $4 \mathrm{~h}$. After incubation medium was removed and $50 \mu \mathrm{l}$ of DMSO was added to dissolve formazan crystals. The absorbance of the samples was measured at $550 \mathrm{~nm}$ with a reference wavelength of $630 \mathrm{~nm}$ on a microplate reader UVM 340 (Biogenet, Poland). The untreated control was normalized to $100 \%$ for each assay, and treatments were expressed as the percentage of control.

\section{Ex vivo skin permeation studies}

Eb permeation from different alginate nanocapsules was studied using pig ear samples obtained from a local slaughterhouse. After thorough washing, the skin was separated from the underlying cartilage and subcutaneous fat, cut and stored at $-20^{\circ} \mathrm{C}$ for up to 1 month after preparation to ensure the skin intergrity. Before use skin samples were thawed to room temperature and hydrated with the $0.05 \mathrm{M}$ phosphate buffer $\mathrm{pH} 7.4$ for 30 min. For the experiment, the skin, cut into proper size was thoroughly mounted and clamped between the donor and receptor compartments of a Franz transdermal diffusion cell set (Hanson Research Corporation, CA, USA). The system was fitted with heater circulator with jacketed vertical glass diffusion cells. The receptor cell was filled with $0.05 \mathrm{M}$ phosphate buffer $\mathrm{pH} 7.4$ maintaining sink conditions and gently stirred with a magnetic stirrer at $350 \mathrm{rpm}$. A total of $1 \mathrm{ml}$ of the Eb-NCS ${ }_{1}, \mathrm{~Eb}_{-} \mathrm{NCS}_{3}$ and Eb-NCS 5 was added in the donor compartment on the stratum corneum side of the skin. The system was adjusted at $32.5^{\circ} \mathrm{C}$. Aliquots $(0.5 \mathrm{ml})$ were withdrawn at specific time intervals $(0.5,1,2,3,4,5,6$ and $24 \mathrm{~h})$ and replaced with fresh medium. All samples were analyzed by HPLC.

For confocal fluorescence experiments, a fluorescent dye, Nile Red, was dissolved in Labrafac Lipophile WL1349 $(0.05 \% \mathrm{w} / \mathrm{w})$ or added during the preparation of the oil phase of $\mathrm{NCS}_{3}(0.05 \% \mathrm{w} / \mathrm{w})$ and the permeation study was performed as described above. The treated skin samples were removed from the Franz diffusion cells after $5 \mathrm{~h}$, washed with distilled water, frozen at $-20^{\circ} \mathrm{C}$, cut and analyzed by confocal laser scanning microscopy (LSM 510 Meta Microscope, Carl Zeiss, Jena, Germany) with a LD Plan-Neofluar $20 \times / 0.4$ objective and DPSS $561 \mathrm{~nm}$ laser.

\section{Ex vivo Eb-NCS: skin interaction study}

Differential scanning calorimetry (DSC) study was performed to find out the interaction of the ebselen-loaded alginate nanocapsules with treated stratum corneum (SC). The pig ear skin samples, with the SC facing up, were 


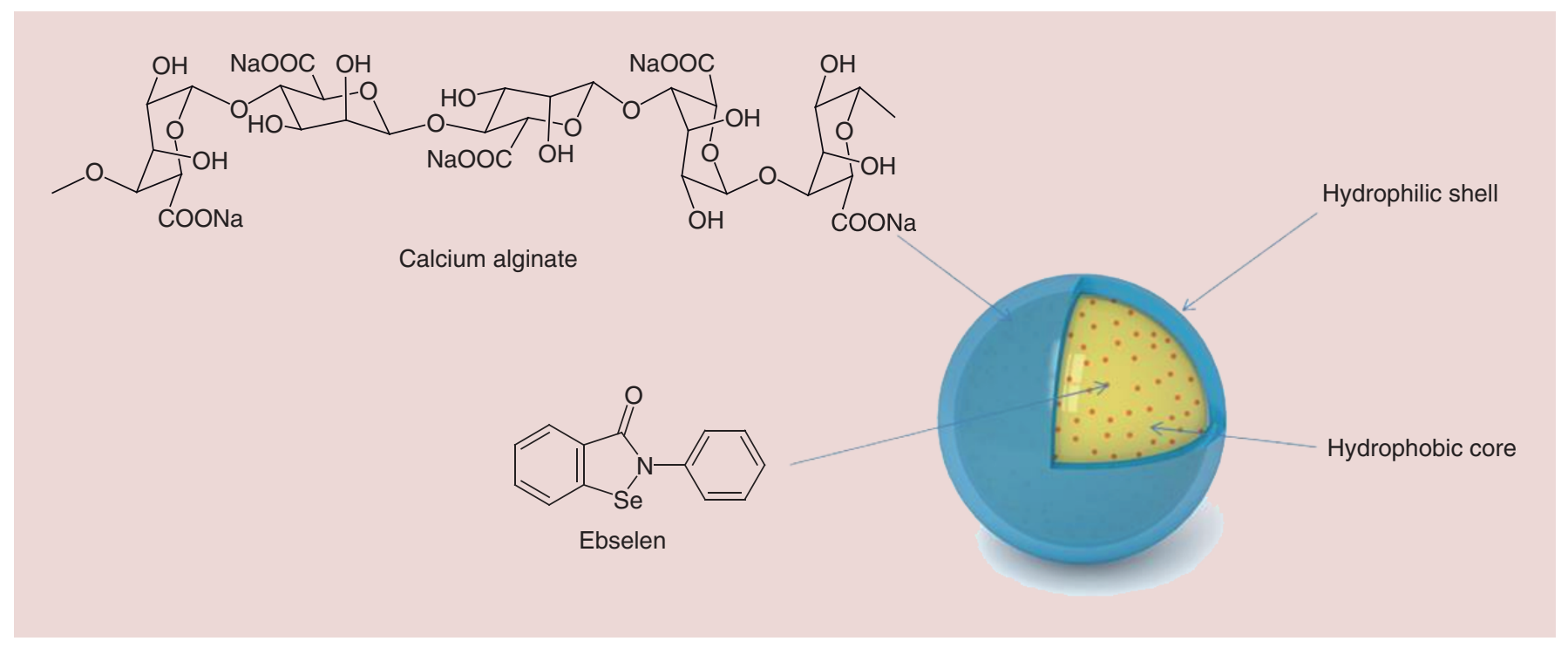

Figure 1. Scheme of ebselen-loaded nanocapsule.

incubated in a petri dish over a Whatman ${ }^{\circledR}$ filter paper saturated with $0.5 \% \mathrm{w} / \mathrm{v}$ aqueous solution of trypsin for $3 \mathrm{~h}$. Then, SC was detached from the rest of the skin and thoroughly washed. Next, SC was treated with $\mathrm{NCS}_{3}$ and Eb-NCS 3 for $12 \mathrm{~h}$, whereas a control sample was prepared in a similar way, without any nanocapsules addition. The SC samples were thoroughly flushed with distilled water, hydrated and dried to obtain 20-40\% hydratation. Each sample of SC from pig skin (about $4.4 \mathrm{mg}$ ) was weighed in aluminum pan, packed tightly and sealed hermetically. The samples were subjected to heat from 25 to $200^{\circ} \mathrm{C}$ at the rate of $10^{\circ} \mathrm{C} / \mathrm{min}$ (DSC1, Mettler-Toledo LLC, OH, USA) in an absolute nitrogen atmosphere $(30 \mathrm{ml} / \mathrm{min})$. The changes in structure of the SC were identified from the obtained thermograms.

\section{Statistical analysis}

All data are expressed as the mean \pm standard deviation of three measurements. Statistical analyses were accomplished using an online one-way analysis of variance with post hoc Tukey honest significant difference calculator [30]. A value of $\mathrm{p}<0.05$ was considered statistically significant.

\section{Results}

\section{Preparation \& characterization of Eb-NCS}

A series of unloaded $\left(\mathrm{NCS}_{1-5}\right)$ and loaded $\left(\mathrm{Eb}-\mathrm{NCS}_{1-5}\right)$ nanocapsules were prepared (Table 1) with different composition of lipophilic core (schematic diagram of the structure is presented in Figure 1). We studied compositions, where the Labrafac Lipophile WL1349 to Transcutol HP ratio was in the range of 5:1-1:1 w/w, whereas a constant level of Span 80 was preserved. All obtained formulations were homogeneous liquids (Figure 2A) with a narrow particle size (Figure 2B) and $\zeta$ potential distribution (Figure 2C). DLS measurements of Eb-loaded nanocapsules showed a mean diameter in the range $172 \pm 1$ to $204 \pm 1 \mathrm{~nm}$ whereas $\zeta$ potential values were $-15.4 \pm 0.1$ to $-17.1 \pm 0.9 \mathrm{mV}$, mainly due to the presence of negatively charged anionic alginate. Moreover, it is commonly believed that values of PdI ranging from 0 to 0.5 characterize monodisperse and homogenous carriers, therefore our results $(0.11 \pm 0.01$ to $0.17 \pm 0.01)$ are very satisfactory. It was found that a decrease in the Labrafac Lipophile WL1349 content followed by a simultaneous increase in the Transcutol HP fraction affected the size and PdI of both $\mathrm{NCS}_{1-5}$ and $\mathrm{Eb}_{-} \mathrm{NC}_{\mathrm{S} 1-5}$. Consequently, nanocapsules with the smallest diameter were obtained in the case of use of $1: 1 \mathrm{w} / \mathrm{w}$ ratio.

To obtain more information about morphology of prepared nanocarries, cryo-TEM, SEM and MALDI-ToF-MS analysis were performed. Microscopic image (Figure 3A) presents sodium alginate-stabilized nanoemulsion before treatment with calcium which is defined as 'intermediate nanoemulsion'. Clearly noticeable less-dense irregular oily core is inside the particles as the brighter phase; which is further hidden after ionic cross-linking of the surface by calcium ions. An efficacious assembly of nanocarrier particles was further confirmed using cryo-TEM imaging 


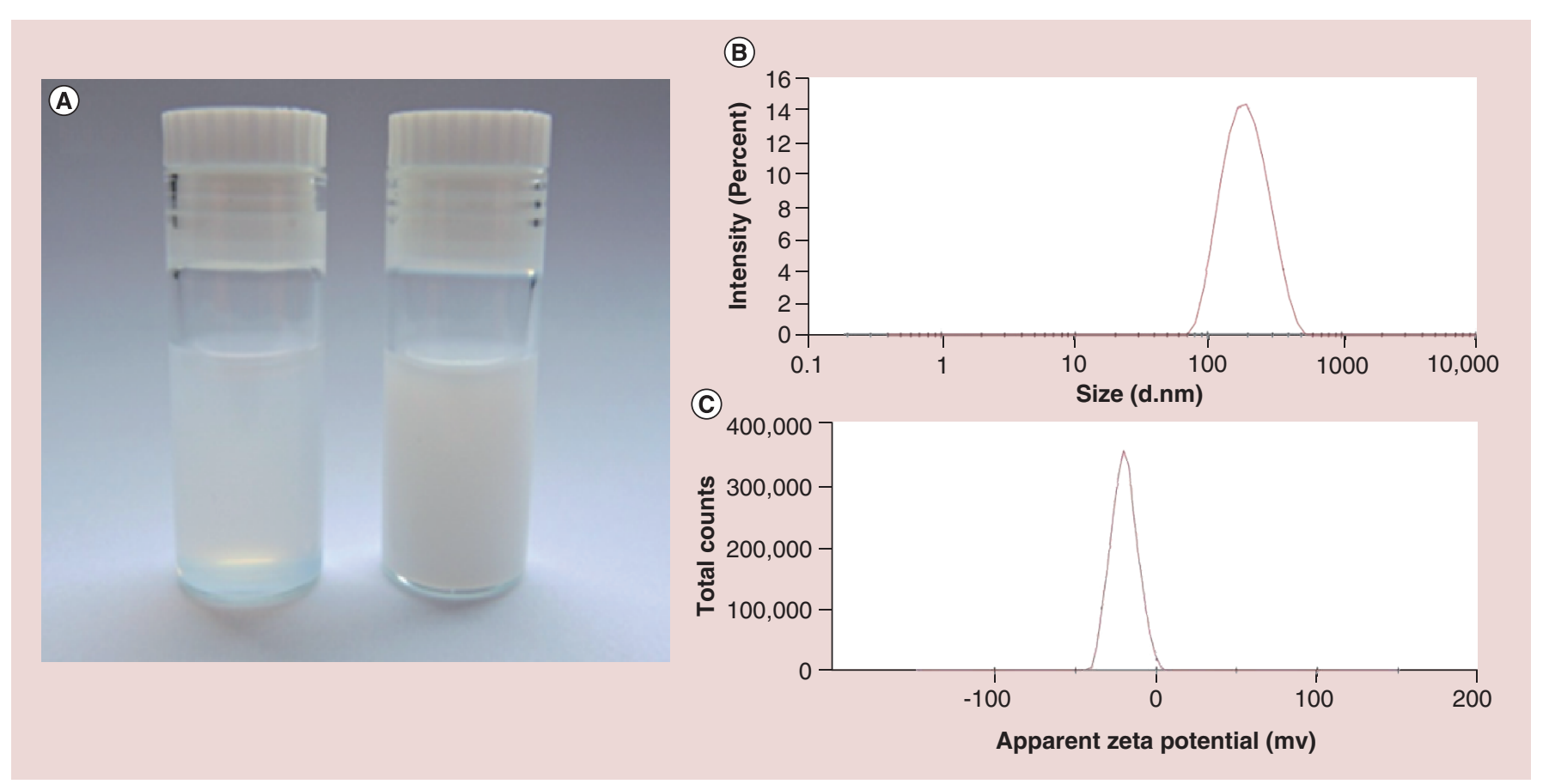

Figure 2. Characteristic of ebselen-loaded nanocapsules. (A) Photograph of Eb-NCS 3 after dilution with distilled water 1:10 v/v (right) and 1:100 v/v (left). (B) Size distribution determined by dynamic light scattering. (C) $\zeta$ potential distribution.

Eb-NC: Ebselen-loaded core-shell nanocapsule.

(Figure 3B) and MALDI-ToF-MS. The latter showed the entire process of nanocapsule formation to be chemically inert as no changes in mass spectra of the individual nanocapsule components were observed (Figure 3E). As no covalent bond-like chemical interactions were determined between both the alginate shell and the in coreencapsulated $\mathrm{Eb}$, this approach further proved the manifestation of a stable separation of core-shell compartments during nanocapsule processing. This core-shell structure of developed nanocapsule with the darker shell and lighter core is presented in Figure 3D. The nanocapsules displayed a fine uniform and spherical shape with good dispersion without aggregation. Moreover, the diameters observed by cryo-TEM and SEM (Figure 3C) were in good agreement with the results of dynamic light scattering measurement. It is worth to mention that there was no evidence of physical Eb precipitation.

The encapsulation efficiencies determined for different types of alginate core-shell carriers were in the range $26.9 \pm 1.2-45.1 \pm 2.1 \%$ (Table 1). It is clear from the data obtained that the ratio of Labrafac Lipophile WL1349 and Transcutol HP in the lipophilic phase has the crucial impact on EE.

Finally, we performed the stability tests which were based on the time dependent changes of size distribution, PdI and $\zeta$ potential of ebselen-loaded nanocapsules. The measurements were performed on the day of preparation and on 7th, 14th and 28th day of storage. Overall, the determined size and PdI was almost constant during experiment, suggesting no tendency to aggregation and fusion phenomenon of ebselen nanocarriers (Figure 4A). The initial $\zeta$ potential of the different nanocapsules were comparable, except Eb-NCS 5 and during storage all values slightly increased (Figure 4B).

Based on above observations, we selected $\mathrm{Eb}_{-} \mathrm{NCS}_{3}$ for further experiments, as model optimal formulation since this composition exhibited satisfactory entrapment efficiency and at the same time high stability.

\section{Antifungal action of Eb-NCS}

Free $\mathrm{Eb}$ showed strong antifungal activity against all four tested Candida species with MIC values ranging from 0.015 to $1.520 \mu \mathrm{g} / \mathrm{ml}$ (Table 2). Comparing to the free drug, the activity of Eb-NCS 3 was significantly increased against three species, C. parapsilosis, C. albicans and C. tropicalis, while the determined MIC values were about 1.25-, 2- and 4-times lower, respectively. No antifungal activity against any of the tested Candida species was observed in the presence of empty nanocapsules without Eb. As shown in Figure 5B, the Eb-NCS 3 tightly attached to the 

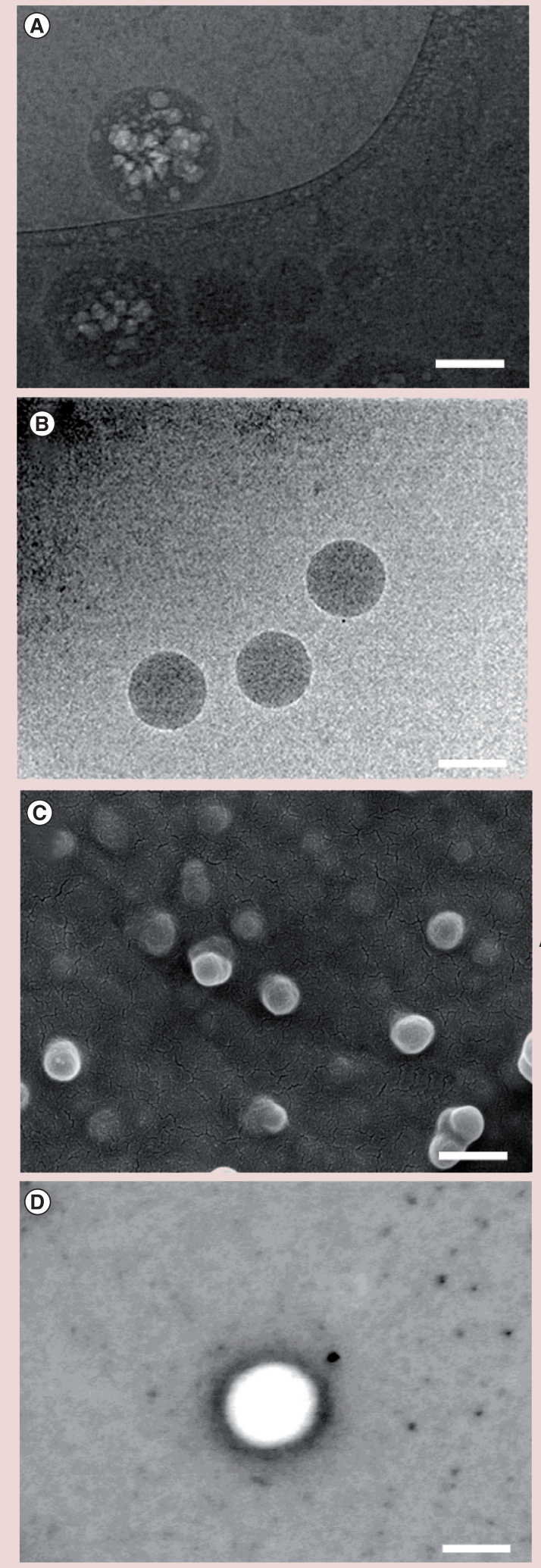

(E)
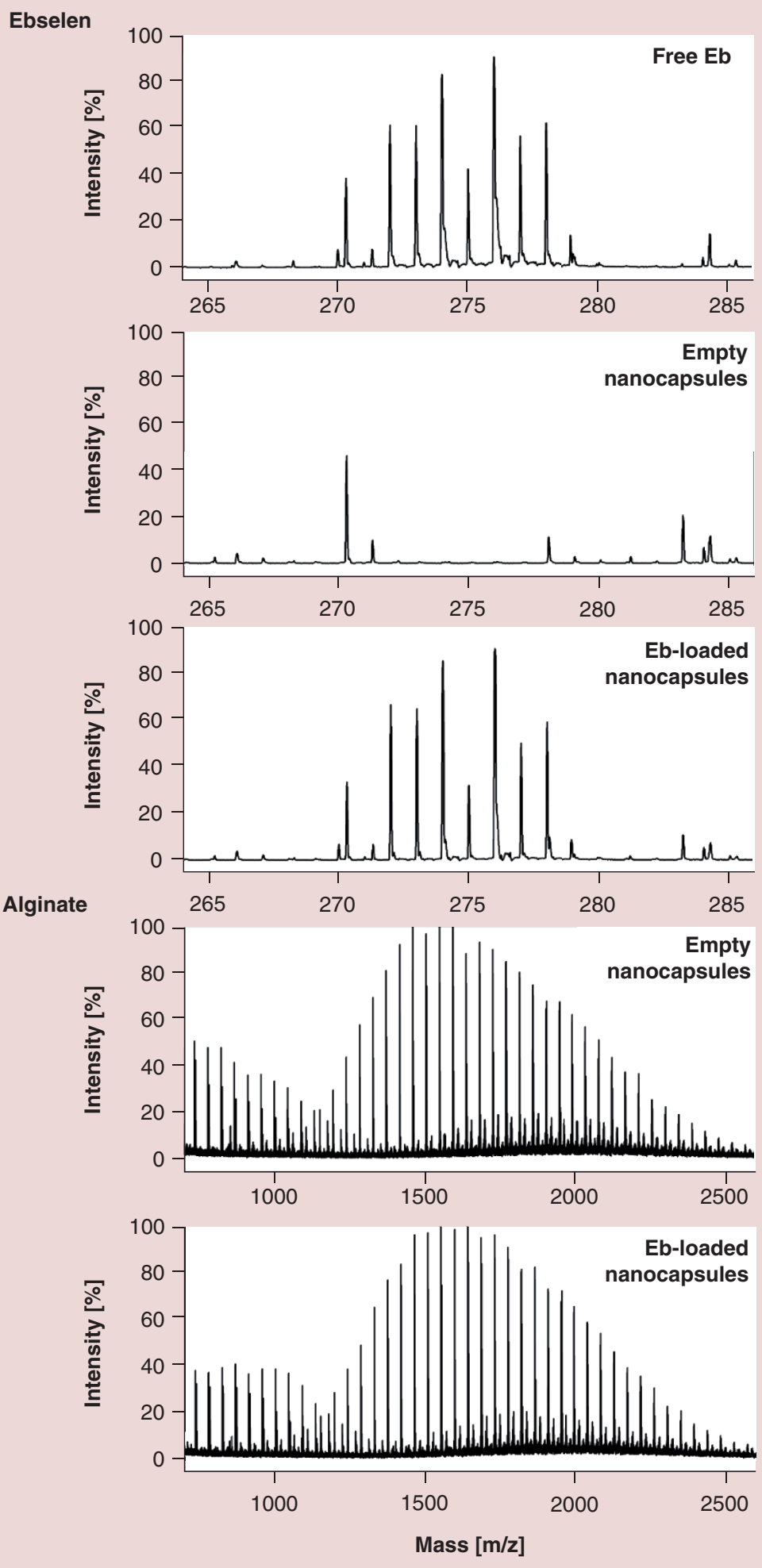

Figure 3. Morphology and analysis of Eb-loaded core-shell nanocapsules. (A) Representative cryo-TEM image of intermediate nanoemulsion. Scale bar: $100 \mathrm{~nm}$. (B) Cryo-TEM visualization of nanocapsules show the presence of uniform spherical shaped structures. Scale bar: $150 \mathrm{~nm}$. (C) SEM image of nanocapsules. Scale bar: $250 \mathrm{~nm}$. (D) SEM image of nanocapsule showing core-shell structure. Scale bar: $100 \mathrm{~nm}$. (E) MALDI-ToF mass spectra of nanocapsule key components, ebselen and alginate, demonstrates inert chemistry of the nanocapsule formation process.

Cryo-TEM: Cryogenic transmission electron microscopy; MALDI ToF: Matrix assisted laser desorption/ionization-time of flight; SEM: Scanning electron microscopy. 


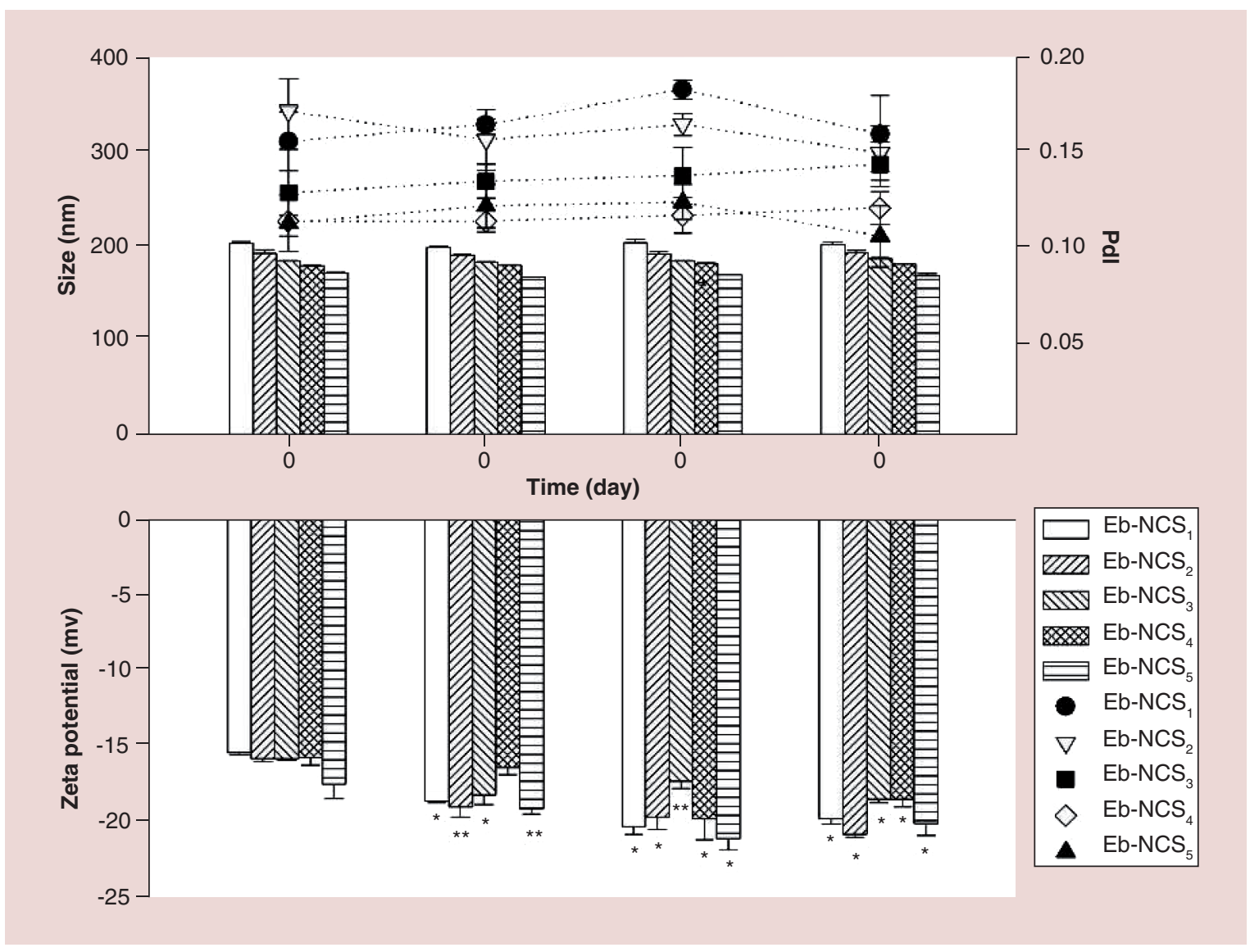

Figure 4. Stability of ebselen-loaded nanocapsules monitored during a 28-day period on storage at room temperature. Mean \pm standard deviation, $n=3,{ }^{*} p<0.01,{ }^{*} \mathrm{p}<0.05$.

Eb-NC: Ebselen-loaded core-shell nanocapsule; Pdl: Polydispersity index.

\begin{tabular}{|c|c|c|}
\hline \multirow[t]{2}{*}{ Candida species } & \multicolumn{2}{|c|}{ MIC $(\mu \mathrm{g} / \mathrm{ml})$} \\
\hline & Free ebselen & Ebselen in Eb-NCS 3 \\
\hline Candida albicans & 0.190 & 0.094 \\
\hline Candida glabrata & 0.024 & 0.094 \\
\hline Candida parapsilosis & 0.015 & 0.012 \\
\hline
\end{tabular}

No growth inhibition of any Candida species was observed for Ebselen-free nanocapsules.

Eb-NC: Ebselen-loaded core-shell nanocapsule; MIC: Minimal inhibitory concentration (the lowest concetration of the tested antifungals that inhibited the visible growth of Candida cells after overnight incubation).

surface of yeast cells of $C$. tropicalis, the most resistant species to the drug in this study. There was no detectable attachment of nanocapsules to cells of C. glabrata (Figure 5A).

Inhibition of oxidation of o/w emulsion by Eb-NCS

We determined that free Eb suppressed lipid peroxidation of flax seed oil emulsion after treatment of inductor $\left(\mathrm{Fe}^{2+}\right)$ in a dose-dependent manner at concentration 1,25 and $50 \mu \mathrm{g} / \mathrm{ml}$, respectively (Figure 6). Higher concentrations of drug ( 25 and $50 \mu \mathrm{g} / \mathrm{ml}$ ) can inhibit in 77.8 and $92.6 \%$ peroxyl radical-induced oxidation of polyunasaturated fatty acids present in the oily part of emulsion. 

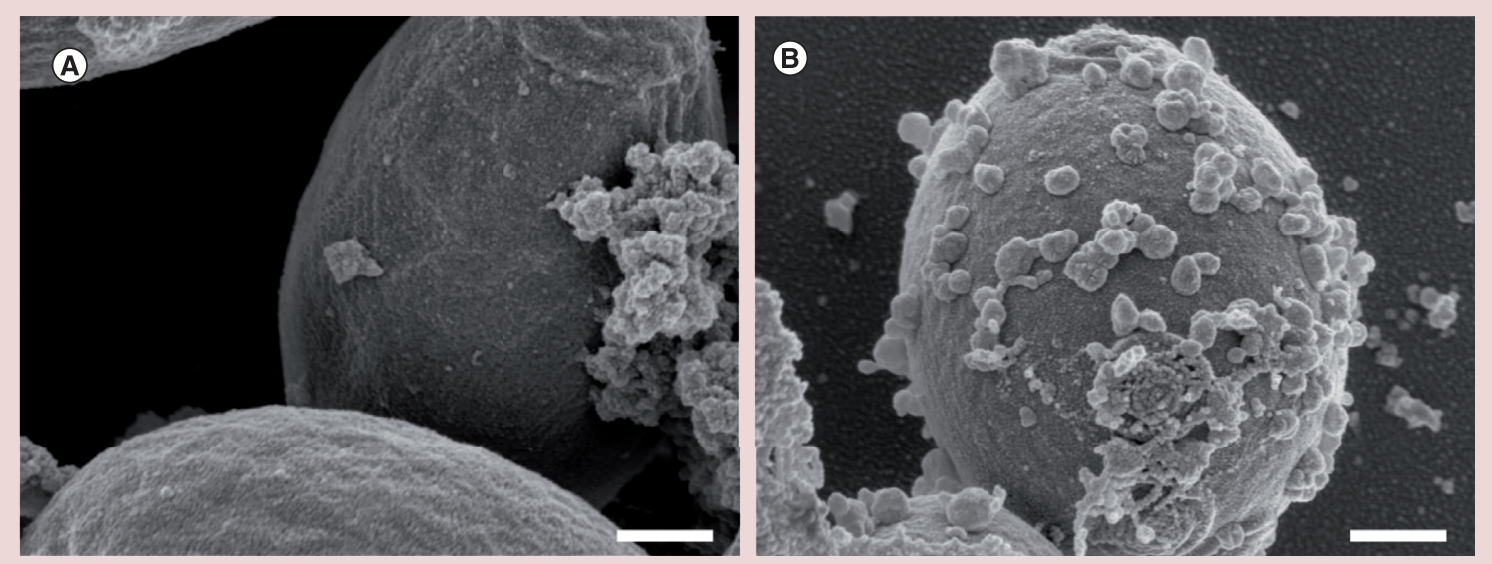

Figure 5. Scanning electron microscopy micrographs showing interactions of Eb-loaded nanocapsules with the cell surface of select Candida spp. yeasts after a 15-min long incubation at room temperature. (A) No detectable attachment of nanocapsules to C. glabrata cells. (B) Tight attachment of nanocapsules to C. tropicalis cells. Scale bars: $400 \mathrm{~nm}$.

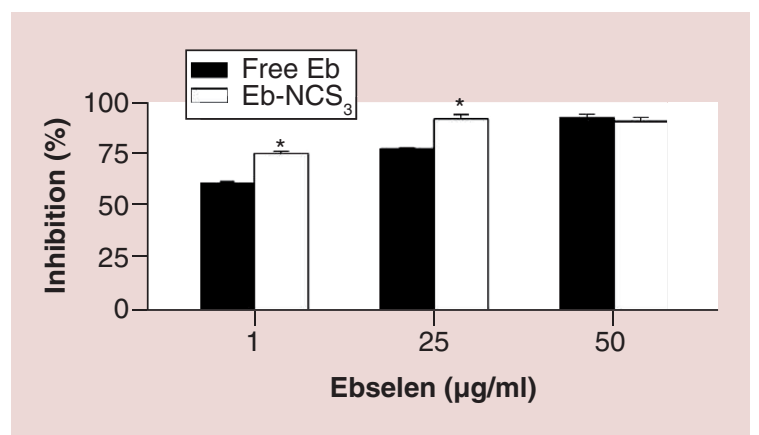

Figure 6. The effect of ebselen and ebselen-loaded nanocapsules on the peroxyl radical-induced oxidation in oil/water emulsion system. Mean \pm standard deviation, $\mathrm{n}=3,{ }^{*} \mathrm{p}<0.01$.

Eb-NC: Ebselen-loaded core-shell nanocapsule.

\section{Cytotoxicity of Eb-NCS on normal human dermal fibroblast cells}

The cytotoxicity, tested on NHDF cells, was investigated following incubation for 24 and 48 h by MTT assay. As a control, we employed untreated cells and free ebselen. All of the cell viability values determined after 24 and $48 \mathrm{~h}$ were higher than $88 \%$ when adding the nanocapsules with encapsulated organoselenium molecule into the cell media (Figure 7A \& B).

\section{Ex vivo skin permeation \& interactions studies}

It was determined that even after $24 \mathrm{~h}$ of contact of Eb-loaded nanocapsules with pig ear skin no presence of $\mathrm{Eb}$ was found in the receptor medium, suggesting that transdermal permeation was not occurred. Figure $8 \mathrm{~A} \& \mathrm{~B}$ show images of confocal microscopy after $5 \mathrm{~h}$ of skin contact with Nile Red dissolved in Labrafac Lipophile WL1349 and Nile Red loaded nanocapsules, respectively. High intensity of fluorescence was detected in upper layer of skin only in the case of nanocapsules, suggesting its retention in SC.

We also carried out DSC analysis to explore thermal transitions in pig SC after contact with nanocapsules. Figure 9 shows DSC thermograms of untreated (control), Eb-NCS 3 and $\mathrm{NCS}_{3}$ treated SC. The untreated SC showed three typical endothermic transition temperatures at $66.63,77.2$ and $118^{\circ} \mathrm{C}$. Addition of Eb-NCS 3 resulted in disapperance of first two peaks whereas the third one was significantly shifted to $103.44^{\circ} \mathrm{C}$.

\section{Discussion}

Drug repositioning, often known as drug repurposing or drug reprofiling, has been growing in importance in the last few years. Our goal in this study was to find enhanced Eb repurposing as an antifungal agent through encapsulation in an appropriate nanosized system dedicated for topical delivery. Therefore, we encapsulated EbNCS using ultrasound oil-in-water emulsification, followed by gelation with calcium ions according to the method 


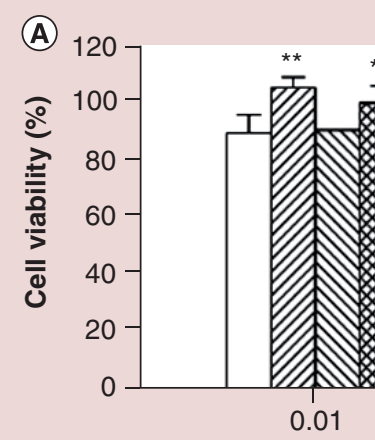

(B)
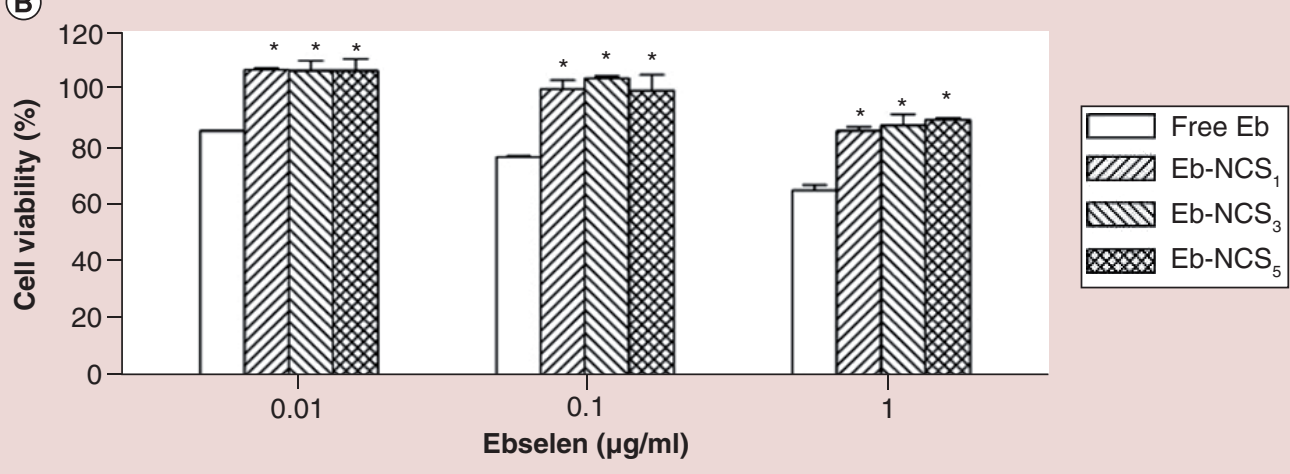

Figure 7. Effects of ebselen and ebselen-loaded nanocapsules on normal human dermal fibroblast cells viability. (A) Cell viability determined after 24 and (B) $48 \mathrm{~h}$. Data were normalized with control (untreated) cell viability (100\%) and expressed as the percentage of the mean \pm standard deviation $(n=3)$, ${ }^{*} p<0.01,{ }^{*} p<0.05$. Eb-NC: Ebselen-loaded core-shell nanocapsule.
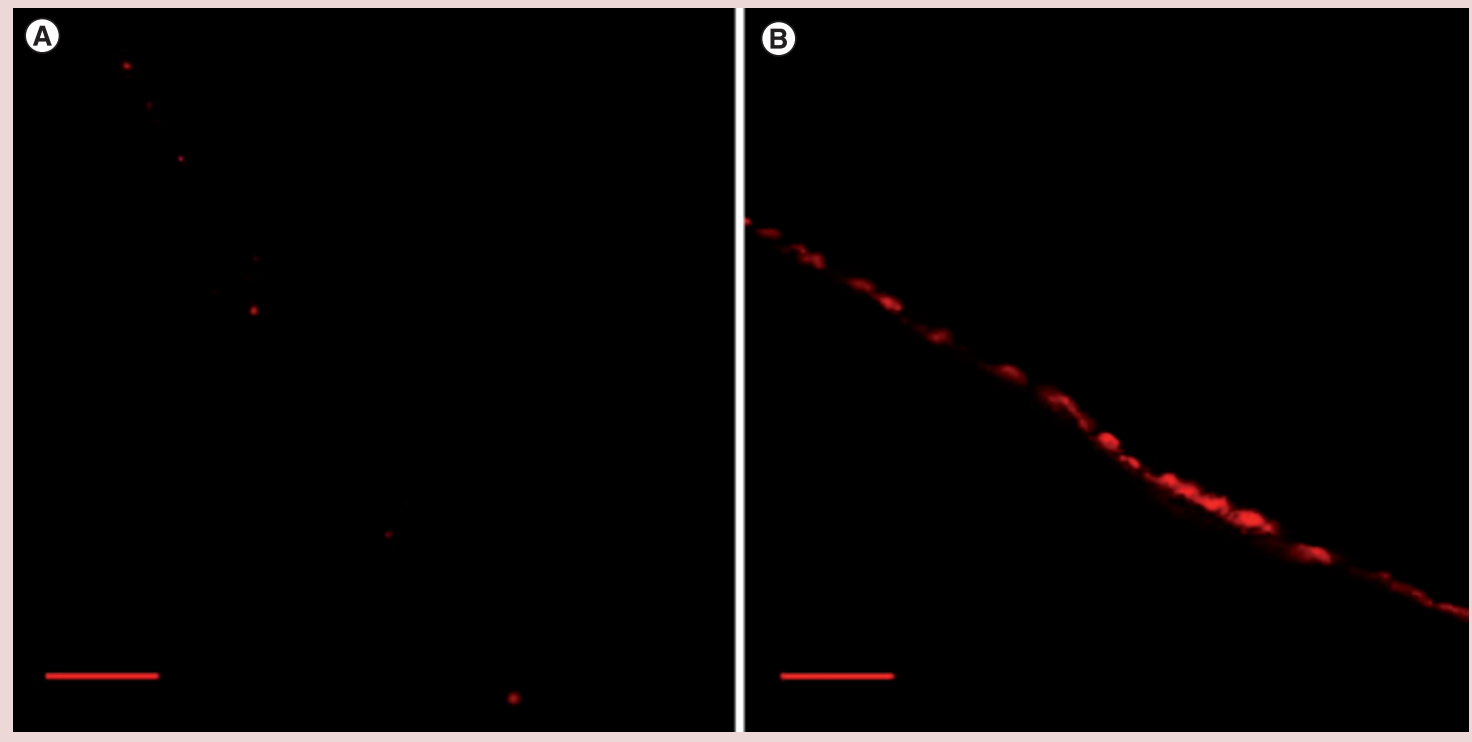

Figure 8. Ex vivo skin permeation demonstrating nanocapsules-skin interactions. Confocal laser scanning microscopy showing distribution of Nile Red in Labrafac Lipophile WL1349 (A) or Nile Red-loaded nanocapsules (B) in pig ear skin after a 5-h-long incubation. Distribution of the encapsulated fluorescent dye only in the stratum corneum layer proves inability of nanocapsules to penetrate deeper intracellular skin spaces. Scale bar represents $100 \mu \mathrm{m}$. 


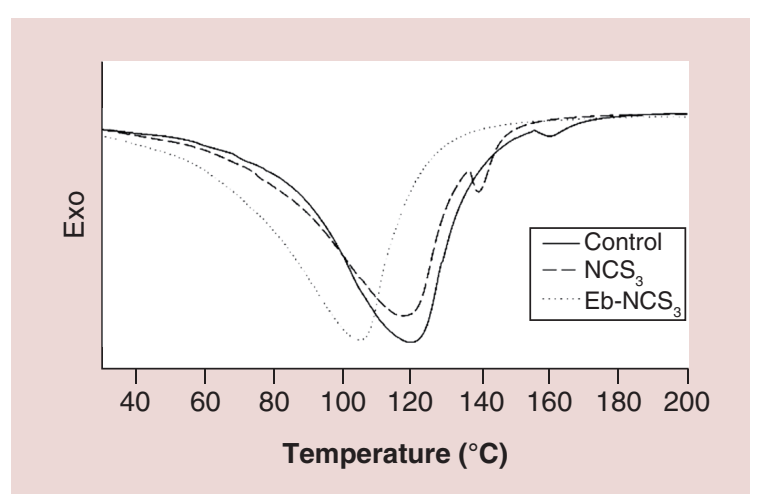

Figure 9. Differential scanning calorimetry thermogram of pig skin stratum corneum treated with the ebselen unloaded and loaded nanocapsules and against the untreated.

Eb-NC: Ebselen-loaded core-shell nanocapsule.

reported by Nguyen $e t$ al. [24]. This robust method allowed successful entrapment of this active compound in the nanosized system at room temperature and without the use of organic solvent. The hydrophilic shell was composed of a polysaccharide material, generally regarded as safe by FDA [31]. Alginate has the property to form hydrogels by spontaneously ionic crosslinking with divalent cations (i.e., $\mathrm{Ca}^{2+}$ ) without no need of temperature or $\mathrm{pH}$ changes [32]. The combination of oily vehicle destined for dissolution of lipophilic ingredients (Labrafac Lipophile WL1349), nonionic surfactant (Span 80) and powerful solvent for poorly water soluble compounds (Transcutol HP) enabled solubilization and encapsulation of our hydrophobic molecule. We were also interested in determination how the changes in composition of the hydrophobic core influenced physicochemical properties and levels of incorporated drug. Interstingly, it was found that the smallest nanocapsules were produced when 1:1 Lipophile WL1349 to Transcutol HP w/w ratio was used. One possible explanation of such an observation is that Transcutol HP, which is miscible in water, escaped from nanocapsule during its formation. As a result, smaller diameters of carriers were obtained when higher levels of this solvent were used. When comparing sizes of $\mathrm{NCS}_{1-5}$ and loaded Eb-NCS ${ }_{1-5}$ we could conclude that in most cases encapsulation of $\mathrm{Eb}$ in core was followed by a slight increase of the size, as a result of its incorporation and to some extent its adsorption in the shell. On the other hand, this process is also accompanied by decrease of PdI, suggesting higher homogeneity of carriers possessing such cargo. Simultaneously, the $\zeta$ potential measurements indicated that the surface charge of nanocapsules was significantly reduced after entrapment of organoselenium molecules.

Based on the determined values of $\log P$ for $\mathrm{Eb}$ - calculated (2.92) [33] and experimentally estimated (2.8) [34] we could conclude that is a hydrophobic molecule. Therefore, predominantly should be present in the oily core of formed nanocapsules. Composition of oily part of nanocapsules enabled the dissolution of Eb, whereas its other physicochemical properties could be hiden by a hydrophilic shell at the same time. Increase of Transcutol HP amount leads to suitable decrease of drug entrapment. Transcutol HP is a small, hydromiscible molecule, thus during production and dialysis process, a part of this solvent leaves probably the inner compartment. This process might cause decreasing of drug level, suggesting simultaneously that Eb has the great affinity to this component. Moreover, we should also consider that some part of Eb could be adsorbed within the alginate shell, as it is suggested for ropivacaine encapsulated in lipid nanocapsules [35]. Such localization could provoke its virtual loss from capsule and subsequently precipitation in aqueous solution. This phenomenon can be also explained by use of calcium chloride to ionically cross-link protective shell of alginate. It is postulated that $\mathrm{CaCl}_{2}$ leads to rapid and poorly controlled gelation due to its high solubility in water [36], thus some leakage of active substance can take place. Problems such as loss of encapsulated material reported for natural based capsules were already described, therefore addition of cationic polymers such as chitosan seems is one of the options [37,38].

Entrapment of organoselenium compound, which is a lipophilic molecule in a core surrounded by hydrophilic alginate shell, ensues its high stability in the aqueous environment. It was found during stability test $\zeta$ potential values increased suggesting stabilization of the system during storage. It is worth noting that the most popular use of $\zeta$ potential data are correlated with colloid stability. Some of the classifications describes nanocarriers with $\zeta$ potential values of $\pm 0-10 \mathrm{mV}, \pm 10-20$ and $\pm 20-30 \mathrm{mV}$ and $> \pm 30 \mathrm{mV}$ as highly unstable, relatively stable, moderately stable and highly stable, respectively [39]. Therefore, based on such assignment, our formulations should be considered as relatively stable. But in the case of nanocapsules Eb-NCS 5 , which showed the smallest size followed by the highest $\zeta$ potential, some precipitates were observed at the end of stability study. This probably is due to a redistribution of $\mathrm{Eb}$ dissolved in Transcutol HP, despite no dramatical changes of physicochemical characteristics 
were detected. Therefore, we adhere to the opinion that the measurements should be always verified by detailed visual observations.

The newly produced Eb loaded system was evaluated for antifungal activity against four most common pathogenic Candida species. The experiments were designed to determine the lowest concentration of $\mathrm{Eb}$ in nanocapsules that inhibited the growth of fungi with reference to free $\mathrm{Eb}$, which was used as control. The measured values were within the range of the data reported recently by Thangamani et al. [13]. Nanocapsules did not exhibit any increased antifungal activity against $C$. glabrata. Such an interesting observation prompted us to perform another experiment which targeted the ability of nanocapsules to interact with and adhere to Candida yeast cells. Interestingly, we observed that no persistent attachment to C. glabrata was detectable, whereas nanacapsules were found tightly attached to $C$. tropicalis cells. This striking phenomenon can be explained due to evolutionary differences between the latter and a group of other tested species, C. parapsilosis, C. albicans and C. tropicalis. In fact, those three species belong to the Candida CTG clade and uniquely translate CTG codons as serine instead of leucine [40], unlike in $C$. glabrata, which utilizes the classical codon. In addition, even within the CTG clade, the genetic distance between individual species is in general significantly large, which is manifested as distinct phenotypic features seen in the biology of those species. We posit that the differences observed in interactions between nanocapsules and tested Candida species were driven by distinct biochemical and biophysical properties of their cell walls.

It is known that $\mathrm{Eb}$ reduces hydroperoxides including hydrogen peroxide, phospholipid hydroperoxide, and cholesterol ester hydroperoxide by GPx mimic action [41-43]. Therefore, we were interested whether our developed system will be able to maintain the antioxidant property of $\mathrm{Eb}$ as it is described for curcumin in alginate nanocarriers [24]. First, we prepared model o/w emulsion containing flaxseed oil, which was incubated with Eb or Eb loaded nanocarriers. After induction of oxidation due to addition $\mathrm{Fe}^{2+}$, the oxidation level was determined by the TBARS assay. However, our findings are opposite with respect to results reported by Hassan et al. [44], who demonstrated that $\mathrm{Eb}$ exerted a small nonsignificant antioxidant effect on $\mathrm{Fe}^{2+}$ induced TBARS production in supernatants of rat brain homogenates. In our case, the effectiveness of $\mathrm{Eb}$ - hydrophobic molecule as antioxidant could be elucidated by polar paradox, the theory that suggests that lipophilic antioxidants are more effective in lipid emulsions [45]. Surprisingly, our studies have also shown that nanocapsules containing this active compound can be even more active than free drug. Core-shell structures with encapsulated Eb protected oxidation more efficiently at concentration 1 and $25 \mu \mathrm{g} / \mathrm{ml}$, resulting in 75.4 and $92.1 \%$ of inhibition, respectively, whereas at the highest concentration this level was comparable. The almost $15 \%$ increase of the observed protection could be explained by synergistic action of drug and alginate. There are several reports demonstrated antioxidant action of alginates from different sources $[46,47]$. Noguchi et al. discovered that Eb suppressed the oxidation of methyl linoleate emulsions in aqueous dispersions induced by iron [48] suggesting that this effect is a result of antioxidant action of drug due to reducing hydroperoxides, which probably takes also place in our system.

Bearing in mind that in vitro tests represent a first step of biomedical application we also investigated the effects of Eb-loaded nanocapsules on viability of normal human dermal fibroblast cells to obtain insight in the potential skin toxicity. Our findings are in well agreement with the recent study, where very similar structures, however without addition of Transcutol HP demonstrated also nonsignificant toxicity on human keratinocytes $(\mathrm{HaCaT})$ cell line even at very high concentration [49]. In our test, we used $\mathrm{Eb}$ concentrations 0.01, 0.1 and $1 \mu \mathrm{g} / \mathrm{ml}$, respectively because in this particular concentration range, inhibitory effects of Eb-NCS 3 on Candida spp. were established. We investigated also Eb-NCS 5 despite its unsatisfactory stability to estimate the effect of oil core composition on cell viability. Interestingly, it was found that all used nanocarriers had almost the same, low toxicities at each concentration. It is worth noting that nanocapsules also exerted protective action, showed higher fibroblast viabilities in comparison to free Eb tested at the same concentrations. This is a result of entrapment of this active compound inside the core of developed system, thus direct contact with cells in culture was seriously limited. Eb is a lipid-soluble compound and can readily enter the cell, which resulted in inhibition of viability observed for free drug. Therefore, our data suggest that the nanocapsules had a good biocompatibility and could be used as drug delivery platform.

In order to evaluate the influence of drug-loaded nanocapsules on skin we performed ex vivo permeation studies. We used pig ear skin because it is often used as a model of human skin due to its histologically similarity [50]. Moreover, pig skin was found to have a closer permeability character than for example, rat skin to human skin, particularly for lipophilic penetrants [51]. Vertical diffusion Franz-type cells and HPLC were used to perform the permeation studies, but Eb was not detected. We therefore sought to perform skin distribution of fluorescent dye, Nile Red incorporated in one of the oil phase component or in nanocapsules. As detected by confocal microscopy 
analysis, a significant accumulation of dye after treatment in encapsulated form was revealed only in the upper layers of the excized skin. In the case of free Nile Red, this phenomenon was very poor. Therefore our findings corroborate the hypothesis [24] that this particular type of carrier is not able to lead to a transdermal delivery of the drug.

These results encouraged us to perform DSC analysis of SC after permeation study of Eb-NCS $\mathrm{N}_{3}$ for $12 \mathrm{~h}$ to detect possible effects on structure of this part of skin, based on thermal properties. The SC is the outermost layer of the epidermis with unique composition of lipids and proteins, responsible for the skin's barrier function. With aid of this technique, we identified interactions between alginate carriers and components of the SC. The untreated SC have three typical endothermic transition temperatures, for the melting of intercellular lipids, proteinassociated lipids, and denatured proteins, respectively [52]. The fact that contact with $\mathrm{Eb}-\mathrm{NCS}_{3}$ caused disapperance of two peaks and shiftment of the third implies that treatment of loaded nanocapsules leads to efficient structural changes of intercellular lipids and proteins. Hence, our results suggested that alginate nanocarriers caused significant modification in architectural structure of SC without ability to transdermal transport.

\section{Conclusion}

This study provides a definitive proof-of-concept of a nanosized drug delivering system for $\mathrm{Eb}$, the molecule receiving a great attention over last several years. The effects of composition of oily part of developed core-shell nanocapsules on size and encapsulation efficiencies were investigated and optimized. Various modern characterization techniques such as cryogenic transmission and SEM, MALDI-ToF-MS were performed for observation of these structures. Results showed that Eb loaded nanocapsules have size $<204 \mathrm{~nm}$, negative potential $\zeta$ and satisfactory PdI. We also found good stability, minimal toxicity toward normal human dermal fibroblasts and inhibition of induced lipid oxidation in the o/w emulsion system. Despite no ex vivo skin permeation was detected, alterations of an organization in SC were recorded. The presence of $\mathrm{Eb}$ in the nanocapsules significantly boosted its antifungal action against most common pathogenic Candida species, which overall makes this newly developed drug delivery nanosystem a promising and host-safe alternative strategy for treatment of cutaneous candidiasis.

\section{Summary points}

- Ebselen-encapsulated core-shell nanocapsules (Eb-NCS) were designed and characterized by different techniques to obtain information about their morphology, size distribution, $\zeta$ potential and encapsulation efficiency.

- Encapsulation of ebselen in nanocapsules improved antifungal drug activity against the tested Candida species of the CTG clade.

- Eb-NCS significantly prevented the oxidation process of flaxseed oil emulsion induced by $\mathrm{Fe}^{2+}$ and showed minimal toxicity toward normal human dermal fibroblasts.

- Skin permeation experiments demonstrated lack of transdermal passage, retention in stratum corneum and the changes in their structure.

- Eb-NCS emerge to be a promising tool for the treatment of cutaneous candidiasis.

\section{Authors' contributions}

A Jaromin conceived the idea and designed the study, conducted the preparation and characterization of nanocapsules, performed the inhibition of oxidation of oil/water emulsion, carried out the ex vivo permeation experiments, analyzed the cytotoxicity, DSC, confocal fluorescence microscopy data and analyzed microscopic images. R Zarnowski designed fungal experiments, MALDI experiments, supervised imaging studies and analyzed microscopic images. M Piętka-Ottlik synthetized ebselen. DR Andes contributed fungal strains and reagents used for fungal analysis and analyzed data obtained from the performed fungal studies. J Gubernator contributed funding and analyzed microscopic images. A Jaromin wrote the paper with contributions from R Zarnowski. All authors agreed on the final version.

\section{Acknowledgements}

The authors would like to acknowledge M Mahmud for his assistance during HPLC analysis, M Zaremba-Czogalla for her support during confocal fluorescence microscopy observations, H Sanchez for his invaluable help with SEM studies, and EG Dominguez for his vital contribution to antifungal testing experiments. This work made use of the EPIC facility of Northwestern University's NUANCE Center, which has received support from the Soft and Hybrid Nanotechnology Experimental (SHyNE) Resource (NSF 
ECCS-1542205); the MRSEC program (NSF DMR-1121262) at the Materials Research Center; the International Institute for Nanotechnology (IIN); the Keck Foundation; and the State of Illinois, through the IIN.

\section{Financial \& competing interests disclosure}

The study was supported by a statutory activity of subsidy from the Polish Ministry of Science and Higher Education for the Faculty of Biotechnology of University of Wroclaw and by the National Science Centre (NCN) under grant no. UMO 2013/09/D/ST5/03814 (synthesis of ebselen, preparation of nanocapsules). Publication costs were supported by Wroclaw Center of Biotechnology program The Leading National Research Center (KNOW) for years 2014-2018. The authors have no other relevant affiliations or financial involvement with any organization or entity with a financial interest in or financial conflict with the subject matter or materials discussed in the manuscript apart from those disclosed.

No writing assistance was utilized in the production of this manuscript.

Ethical conduct of research

The authors state that they have obtained appropriate institutional review board approval or have followed the principles outlined in the Declaration of Helsinki for all human or animal experimental investigations. In addition, for investigations involving human subjects, informed consent has been obtained from the participants involved.

\section{Open access}

This work is licensed under the Attribution-NonCommercial-NoDerivatives 4.0 Unported License. To view a copy of this license, visit http://creativecommons.org/licenses/by-nc-nd/4.0/

\section{References}

Papers of special note have been highlighted as: $\bullet$ of interest; $\bullet \bullet$ of considerable interest

1. Azad GK, Tomar RS. Ebselen, a promising antioxidant drug: mechanisms of action and targets of biological pathways. Mol. Biol. Rep. 41(8), 4865-4879 (2014).

- Summary of the biochemical and molecular properties of ebselen and its pharmacological applications.

2. Nakamura $Y$, Feng Q, Kumagai T et al. Ebselen, a glutathione peroxidase mimetic seleno-organic compound, as a multifunctional antioxidant. Implication for inflammation-associated carcinogenesis. J. Biol. Chem. 277(4), 2687-2694 (2002).

3. Schewe T. Molecular actions of ebselen - an anti-inflammatory antioxidant. Gen Pharmacol. 26(6), 1153-1169 (1995).

4. Chew P, Yuen DY, Stefanovic $\mathrm{N}$ et al. Antiatherosclerotic and renoprotective effects of ebselen in the diabetic apolipoprotein E/GPx1-double knockout mouse. Diabetes 59(12), 3198-3207 (2010).

5. Singh N, Sharpley AL, Emir UE et al. Effect of the putative lithium mimetic ebselen on brain myo-inositol, sleep, and emotional processing in humans. Neuropsychopharmacology 41(7), 1768-1778 (2016).

6. Masaki C, Sharpley AL, Cooper CM et al. Effects of the potential lithium-mimetic, ebselen, on impulsivity and emotional processing. Psychopharmacology (Berl.) 233(14), 2655-2661 (2016).

7. Thenin-Houssier S, De Vera IM, Pedro-Rosa L et al. Ebselen, a small-molecule capsid inhibitor of HIV-1 replication. Antimicrob. Agents Chemother. 60(4), 2195-2208 (2016).

8. Favrot L, Grzegorzewicz AE, Lajiness DH et al. Mechanism of inhibition of Mycobacterium tuberculosis antigen 85 by ebselen. Nat. Commun. 4, 2748 (2013).

9. Nozawa R, Yokota T, Fujimoto T. Susceptibility of methicillin-resistant Staphylococcus aureus to the selenium-containing compound 2-phenyl-1,2-benzoisoselenazol-3(2H)-one (PZ51). Antimicrob. Agents Chemother. 33(8), 1388-1390 (1989).

10. Thangamani S, Mohammad H, Younis W, Seleem MN. Drug repurposing for the treatment of staphylococcal infections. Curr. Pharm. Des. 21(16), 2089-2100 (2015).

11. Thangamani S, Younis W, Seleem MN. Repurposing clinical molecule ebselen to combat drug resistant pathogens. PLoS ONE 10(7), e0133877 (2015).

- Describes the potential use of ebselen in treatment of multidrug resistant Gram-positive bacterial infections alone or in combination with other antibiotics.

12. Younis W, Thangamani S, Seleem MN. Repurposing non-antimicrobial drugs and clinical molecules to treat bacterial infections. Curr. Pharm. Des. 21(28), 4106-4111 (2015).

13. Thangamani S, Eldesouky HE, Mohammad H et al. Ebselen exerts antifungal activity by regulating glutathione (GSH) and reactive oxygen species (ROS) production in fungal cells. Biochim. Biophys. Acta 1861(1 Pt A), 3002-3010 (2017).

14. Kuhbacher A, Burger-Kentischer A, Rupp S. Interaction of Candida species with the skin. Microorganisms 5(2), pii:E32 (2017).

- Discusses complex commensal and infectious aspects of Candida biology growing on the skin tissue. 
15. Perlin DS, Rautemaa-Richardson R, Alastruey-Izquierdo A. The global problem of antifungal resistance: prevalence, mechanisms, and management. Lancet Infect. Dis. 17(12), E383-E392 (2017).

-• Describes current challenges in the field of infectious disease as a result of emerging antifungal drug resistance worldwide.

16. Nosengo N. Can you teach old drugs new tricks? Nature 534(7607), 314-316 (2016).

17. Singh N, Halliday AC, Thomas JM et al. A safe lithium mimetic for bipolar disorder. Nat. Commun. 4, 1332 (2013).

18. Guisbiers G, Wang Q, Khachatryan $\mathrm{E}$ et al. Inhibition of $E$. coli and $S$. aureus with selenium nanoparticles synthesized by pulsed laser ablation in deionized water. Int. J. Nanomedicine 11, 3731-3736 (2016).

19. Piętka-Ottlik M, Lewińska A, Jaromin A, Krasowska A, Wilk KA. Antifungal organoselenium compound loaded nanoemulsions stabilized by bifunctional cationic surfactants. Colloids Surf. A. Physicochem. Eng. Asp. 510(Suppl. C), 53-62 (2016).

20. Wang Q, Larese-Casanova P, Webster TJ. Inhibition of various Gram-positive and Gram-negative bacteria growth on selenium nanoparticle coated paper towels. Int. J. Nanomedicine 10, 2885-2894 (2015).

21. Eral HB, López-Mejías V, O'mahony M, Trout BL, Myerson AS, Doyle PS. Biocompatible alginate microgel particles as heteronucleants and encapsulating vehicles for hydrophilic and hydrophobic drugs. Cryst. Growth Des. 14(4), 2073-2082 (2014).

22. Jain D, Bar-Shalom D. Alginate drug delivery systems: application in context of pharmaceutical and biomedical research. Drug Dev. Ind. Pharm. 40(12), 1576-1584 (2014).

23. Pietka-Ottlik M, Wojtowicz-Mlochowska H, Kolodziejczyk K, Piasecki E, Mlochowski J. New organoselenium compounds active against pathogenic bacteria, fungi and viruses. Chem. Pharm. Bull. (Tokyo) 56(10), 1423-1427 (2008).

24. Nguyen HT, Munnier E, Souce $M$ et al. Novel alginate-based nanocarriers as a strategy to include high concentrations of hydrophobic compounds in hydrogels for topical application. Nanotechnology 26(25), 255101 (2015).

-. Describes the method of preparation of carriers for encapsulation of hydrophobic active molecules for topical delivery.

25. Institute CaLS. Performance standards for antimicrobial susceptibility testing. In: Twenty-Fifth Informational Supplement. CLSI document M100-S25 (2015). https://clsi.org/standards/products/microbiology/documents/m100/

26. Jaromin A, Zarnowski R, Kozubek A. Emulsions of oil from Adenanthera pavonina L. seeds and their protective effect. Cell. Mol. Biol. Lett. 11(3), 438-448 (2006).

27. Zarnowski R, Jaromin A, Certik M et al. The oil of Adenanthera pavonina L. seeds and its emulsions. Z. Naturforsch. C. 59(5-6), 321-326 (2004).

28. Buege JA, Aust SD. Microsomal lipid peroxidation. Methods Enzymol. 52, 302-310 (1978).

29. Mosmann T. Rapid colorimetric assay for cellular growth and survival: application to proliferation and cytotoxicity assays. J. Immunol. Methods 65(1-2), 55-63 (1983).

30. ANOVA with post hoc Tukey HSD calculator. http://astatsa.com/OneWay_Anova_with_TukeyHSD/

31. George M, Abraham TE. Polyionic hydrocolloids for the intestinal delivery of protein drugs: alginate and chitosan - a review. J. Control Release 114(1), 1-14 (2006).

32. Grant GT, Morris ER, Rees DA, Smith PJC, Thom D. Biological interactions between polysaccharides and divalent cations: the egg-box model. FEBS Letters 32(1), 195-198 (1973).

33. Pino MA, Pietka-Ottlik M, Billack B. Ebselen analogues reduce 2-chloroethyl ethyl sulphide toxicity in A-431 cells. Arh. Hig. Rada. Toksikol. 64(1), 77-86 (2013).

34. Filipovska A, Kelso GF, Brown SE, Beer SM, Smith RA, Murphy MP. Synthesis and characterization of a triphenylphosphonium-conjugated peroxidase mimetic. Insights into the interaction of ebselen with mitochondria. J. Biol. Chem. 280(25), 24113-24126 (2005).

35. Zhai Y, Yang X, Zhao L, Wang Z, Zhai G. Lipid nanocapsules for transdermal delivery of ropivacaine: in vitro and in vivo evaluation. Int. J. Pharm. 471(1-2), 103-111 (2014).

36. Lee KY, Mooney DJ. Alginate: properties and biomedical applications. Prog. Polym. Sci. 37(1), 106-126 (2012).

37. Lertsutthiwong P, Rojsitthisak P, Nimmannit U. Preparation of turmeric oil-loaded chitosan-alginate biopolymeric nanocapsules. Mater. Sci. Eng. C 29(3), 856-860 (2009).

38. Vandenberg GW, Drolet C, Scott SL, De La Noue J. Factors affecting protein release from alginate-chitosan coacervate microcapsules during production and gastric/intestinal simulation. J. Control. Rel. 77(3), 297-307 (2001).

39. Patel VR, Agrawal YK. Nanosuspension: an approach to enhance solubility of drugs. J. Adv. Pharm. Technol. Res. 2(2), 81-87 (2011).

40. Papon N, Courdavault V, Clastre M, Bennett RJ. Emerging and emerged pathogenic Candida species: beyond the Candida albicans paradigm. PLoS Pathog. 9(9), e1003550 (2013).

-• Thorough description of pathobiology and genetics of clinically important emering and emerged Candida species.

41. Maiorino M, Roveri A, Ursini F. Antioxidant effect of Ebselen (PZ 51): peroxidase mimetic activity on phospholipid and cholesterol hydroperoxides vs free radical scavenger activity. Arch. Biochem. Biophys. 295(2), 404-409 (1992). 
42. Noguchi N, Gotoh N, Niki E. Effects of ebselen and probucol on oxidative modifications of lipid and protein of low density lipoprotein induced by free radicals. Biochim. Biophys. Acta 1213(2), 176-182 (1994).

43. Sattler W, Maiorino M, Stocker R. Reduction of HDL- and LDL-associated cholesterylester and phospholipid hydroperoxides by phospholipid hydroperoxide glutathione peroxidase and Ebselen (PZ 51). Arch. Biochem. Biophys. 309(2), 214-221 (1994).

44. Hassan W, Ibrahim M, Nogueira CW et al. Enhancement of iron-catalyzed lipid peroxidation by acidosis in brain homogenate: comparative effect of diphenyl diselenide and ebselen. Brain Res. 1258, 71-77 (2009).

45. Frankel EN. Antioxidants in lipid foods and their impact on food quality. Food Chem. 57(1), 51-55 (1996).

46. Kelishomi ZH, Goliaei B, Mahdavi $\mathrm{H}$ et al. Antioxidant activity of low molecular weight alginate produced by thermal treatment. Food Chem. 196, 897-902 (2016).

47. Sellimi S, Younes I, Ayed HB et al. Structural, physicochemical and antioxidant properties of sodium alginate isolated from a Tunisian brown seaweed. Int. J. Biol. Macromol. 72, 1358-1367 (2015).

48. Noguchi N, Yoshida Y, Kaneda H, Yamamoto Y, Niki E. Action of ebselen as an antioxidant against lipid peroxidation. Biochem. Pharmacol. 44(1), 39-44 (1992).

49. Nguyen HT, Allard-Vannier E, Gaillard C et al. On the interaction of alginate-based core-shell nanocarriers with keratinocytes in vitro. Colloids Surf. B Biointerfaces 142, 272-280 (2016).

50. Gray GM, Yardley HJ. Lipid compositions of cells isolated from pig, human, and rat epidermis. J. Lipid Res. 16(6), 434-440 (1975).

51. Dick IP, Scott RC. Pig ear skin as an in vitro model for human skin permeability. J. Pharm. Pharmacol. 44(8), 640-645 (1992).

52. Golden GM, Guzek DB, Harris RR, Mckie JE, Potts RO. Lipid thermotropic transitions in human stratum corneum. J. Invest. Dermatol. 86(3), 255-259 (1986). 